\title{
TEMPORAL LOBE EPILEPSY AND PSYCHOSIS
}

$\underline{\text { Núria Santos }}{ }^{1} ;$ António Alho $^{1}$; André Ribeirinho ${ }^{1}$; Liliana Ferreira ${ }^{1}$; Marisa Martins ${ }^{1}$; Nuno Fernandes ${ }^{1}$; Ricardo Gasparinho ${ }^{1}$; Elisabete

Sêco ${ }^{1}$

1- Department of Psychiatry and Mental Health, Hospital Distrital de Santarém, Portugal

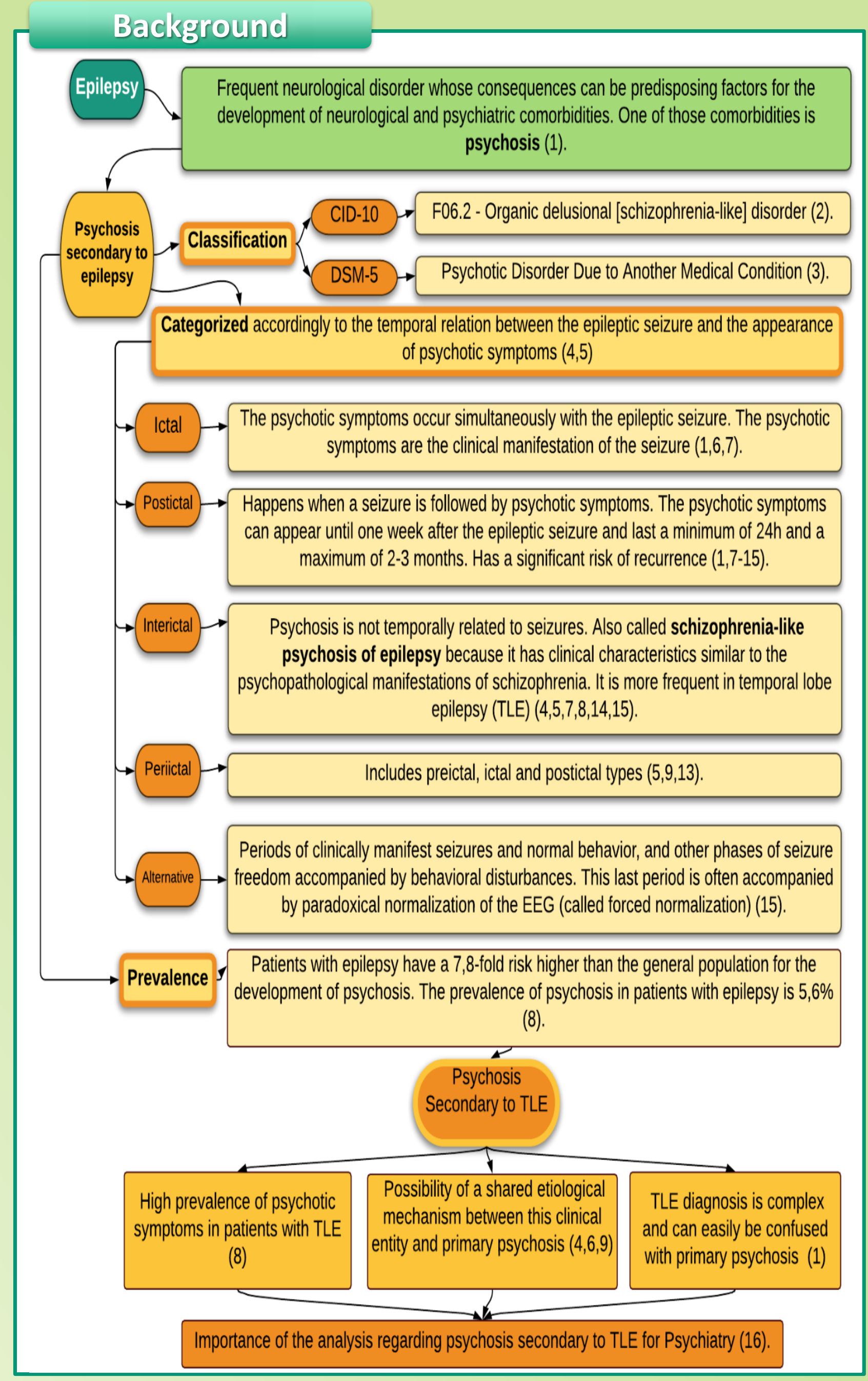

Objectives

- To review psychosis secondary to temporal lobe epilepsy (TLE), focusing on pathogenesis, diagnostic challenges and treatment difficulties.

\section{Methods}

- Literature research conducted using "PubMed" database and search equation built using the MeSH terms "Schizophrenia Spectrum and Other Psychotic Disorders" AND "Epilepsy, Temporal Lobe", restricted to articles written in English or Portuguese, over the last 15 years. Total of 83 results; 59 articles excluded.

\section{Results}

Aetiology

- Limbic system dysfunction seems to be a risk factor for psychosis in TLE (9).

- Patients with epilepsy have a 2 to 3 -fold higher risk of developing schizophrenia than the general population $(5,17,18)$. This higher prevalence of psychotic symptoms in patients with epilepsy suggests a common aetiology between schizophrenia and epilepsy $(4,6,19)$ : neurotoxic effects of epilepsy $\rightarrow$ insidious structure damage $\rightarrow$ increased time between the seizures and the appearance of psychotic symptoms (4).

Diagnosis

- There are main differences between schizophrenia and psychosis secondary to TLE (20). Psychosis in TLE is characterized by: proper mood and affects; infrequent cognitive decline (4-6); uncommon negative symptoms; better premorbid functioning $(5,21)$; common visual ( $20 \%$ of the cases) and olfactive hallucinations $(4,6,22)$; insight; frequent religious delusions $\Rightarrow$ sometimes these differences are faint, with overlapping symptoms between schizophrenia and psychosis in TLE (4).

- Complementary diagnostic tests seem to have limited value $(5,7)$. Example: a retrospective study (6) found no significant associations between acute psychosis and abnormal electroencephalogram (EEG). They concluded there was no indication for routine use of EEG in patients presenting with acute psychosis.

\section{Treatment}

- Currently, there are no clearly defined therapeutic protocols.

General therapeutic recommendations state:

$\Rightarrow$ Ictal: demands a tight control of the epileptic seizure using anticonvulsant medications (5).

$\Rightarrow$ Postictal: treatment requires antipsychotic medication, with a slow weaning off period (23).

$\Rightarrow$ Interictal: treatment also requires antipsychotic medication, but the therapeutic strategies are similar to those used in primary psychosis. Treatment should be maintained after the psychotic symptoms' remission $(5,23)$.

$\Rightarrow$ Alternative: recommendations of the International League Against Epilepsy $(23,24,25)$ are sparce. They state that a decision requires a multidisciplinary team.

$\Rightarrow$ Antipsychotic agents vs Anticonvulsants: atypical antipsychotics seem to be the most appropriate for the treatment of psychotic symptoms in TLE $\rightarrow$ less epileptogenic profile (26). Antipsychotic agents can induce epileptic seizures between $0,1 \%$ and $1,5 \%$ of cases, but the patients also medicated with anticonvulsant drugs have a really low risk of having an epileptic seizure (5) $\rightarrow$ risks and benefits of antipsychotic and anticonvulsant agents must be considered altogether when stablishing the therapeutic protocol.

\section{Conclusion}

- Scientific date about psychosis secondary to TLE is scarce. In the future, the clarification of the relationship between of TLE and the development of psychotic symptoms may contribute to the explanation of the aetiology and pathophysiology of psychosis, as well as for the development of diagnostic strategies and specific therapy for this disorder.

\section{Bibliography}

1. Irwin LG, Fortune DG. Risk Factors for Psychosis Secondary to Temporal Lobe Epilepsy: A Systematic Review. J Neuropsychiatry Clin Neurosci. 2014;26(1):5-23. 2. Organization WH. F00-F09: Organic, including symptomatic, mental disorders. In: Internationa Classification of Diseases - 10th revision. Geneva: World Health Organization; 2016. 3. American Psychiatric Association. Diagnostic and Statistical Manual of Mental Disorders: 5th edition. Washington, DC: American Psychiatric Publishing; 2013. 4. Kandratavicius L, Hallak JE, Leite JP. What are the similarities and differences between schizophrenia and schizophrenia-like psychosis of epilepsy? A neuropathological approach to the understanding of schizophrenia spectrum and epilepsy. Epilepsy Behav. Elsevier Inc.; 2014;38:143-7. 5. Ellio With Acute Psychosis. J Neuropsychiatry Clin Neurosci. 2012;244(4):452-7. 7. Butler T, Weisholtz D, Isenberg N, Harding E, Epstein J, Stern E, et al. Neuroimaging of frontal-limbic dysfunction in schizophrenia and epilepsy-related psychosis: Toward a conat neurobiology. Epilepsy Behav. 2012;23(2):113-22. 8. Clancy MI, Clarke MC, Connor DJ, Cannon M, Cotter DR. The prevalence of psychosis in epilepsy; a systematic review and meta-analysis. BMC Psychiatry. 2014;14(75):1-9.9. Kandratavicius L, Lopes-Aguiar C, Bueno-

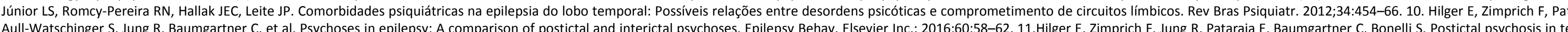
Aull-Watschinger S, Jung R, Baumgartner C, et al. Psychoses in epilepsy: A comparison of postictal and interictal psychoses. Epilepsy Behav. Elsevier Inc.; 2016;60:58-62. 11. Hilger E, Zimprich F, Jung R, Pataraia E, Baumgartner C, Bonelli S. Postictal psychosis in tempo
lobe epilepsy: A case-control study. Eur J Neurol. 2013;1-7. 12. Cleary RA, Thompson PJ, Thom M, Foong J. Postictal psychosis in temporal lobe epilepsy: Risk factors and postsurgical outcome? Epilepsy Res. 2013;106:264-72. 13.González Mingot C, Gil Villar MP, Calvo

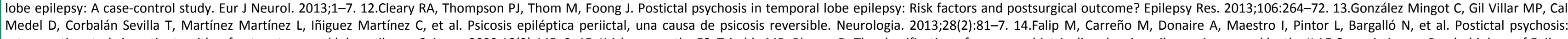
retrospective study in patients with refractory temporal lobe epilepsy. Seizure. 2009;18(2):145-9. 15. Krishnamoorthy ES, Trimble MR, Blumer D. The classification of neuropsychiatric disorders in epilepsy: A proposal by the ILAE Commission on Psychobiology of Epileps JP. Differential aberrant sprouting in temporal lobe epilepsy with psychiatric co-morbidities. Psychiatry Res [Internet]. Elsevier Ltd; 2012;195(3):144-50. 18. Kandratavicius L, Peixoto-Santos JE, Monteiro MR, Scandiuzzi RC, Carlotti CG, Assirati JA, et al. Mesial tempo
lobe epilepsy with psychiatric comorbidities: A place for differential neuroinflammatory interplay. J Neuroinflammation. 2015;12(38):1-12. 19. Tebartz Van Elst L, Baeumer D, Lemieux L, Woermann FG, Koepp M, Krishnamoorthy S, et al. Amygdala pathology in psycho of epilepsy: A magnetic resonance imaging study in patients with temporal lobe epilepsy. Brain. 2002;125:140-9. 20. Slater E, Beard AW. The Schizophrenia-like Psychoses of Epilepsy i. Psychiatric Aspects. Br J Psychiatry. 1963;109:95-150. 21. Needham E, Hamelijnck J. Temporal lobe epilepsy masquerading as psychosis - a case report and literature review. Neurocase. 2012;18(5):400-4. 22. Gandhi P, Ogunyemi B, MacDonald A, Gadit A. Psychosis in temporal lobe epilepsy: Atypical presentation. BMJ Case Rep. 2012;1-3. 23. Kerr MP, Mensah S, Besag F, Toffol B De, Ettinger A, Kanemoto K, et al. International consensus clinical practice statements for the treatment of neuropsychiatric conditins aassociated with epilepsy. Epilepsia. 2018;52:2133-8. 24. Fisher RS, Cross JH, French JA, Higurashin, Pelto 